\title{
Low-frequency Raman Spectroscopy of Aqueous Solutions of Aliphatic Alcohols
}

\author{
Koji Yoshida and Toshio Yamaguchi \\ Department of Chemistry, Faculty of Science, Fukuoka University, \\ Jonan-ku, Fukuoka 814-0180, Japan \\ Reprint requests to Prof. T. Y; Fax : +81-92-865-6030; \\ e-mail : yamaguch@sunsp1.sc.fukuoka-u.ac.jp
}

Z. Naturforsch. 56 a, 529-536 (2001); received May 28, 2001

\begin{abstract}
Low-frequency Raman spectra have been measured at room temperature as functions of the alcohol mole fraction in aqueous solutions of methanol, ethanol, 1-propanol, 2-propanol, and tert-butylalcohol (TBA). Intrinsic Raman spectra $R(\bar{\nu})$ were obtained from depolarized Rayleigh wing spectra. Isosbestic points have been observed in $R(\bar{\nu})$ of the aqueous solutions of ethanol, 1-propanol, and 2-propanol, suggesting that the structure of the solutions is characterized by individual alcohol aggregates and water clusters without a significant amount of alcohol-water mixed aggregates. The $R(\bar{\nu})$ spectra have been expressed as $R(\bar{\nu}, x)=w R(\bar{\nu}, 0)+a R(\bar{\nu}, 1)$, where $R(\bar{\nu}, 0)$ and $R(\bar{\nu}, 1)$ are those for pure water and pure alcohols, respectively, and $x$ is the mole fraction of alcohols. The coefficients $w$ and $a$ show the inflection points at characteristic alcohol mole fractions, where microhetrogeneity and structural transition of the solvent clusters take place, as previously shown by X-ray diffraction. In the aqueous solutions of methanol, where no microhetrogeneity takes place, no clear isosbestic point in $R(\bar{\nu})$ has been observed. For aqueous solutions of TBA, an isosbestic point in $R(\bar{\nu})$ has appeared when $x_{\mathrm{TBA}}>0.05$. Two inflections points in the coefficients have been observed at $x_{\mathrm{TBA}} \approx 0.1$ and 0.35 ; the former composition corresponds to the transition composition from the TBA-TBA intermolecular contact to the TBAwater molecular association, as previously reported by neutron diffraction.
\end{abstract}

Key words: Low Frequency Raman Spectroscopy; Liquid Structure; Alcohol-water Mixture.

\section{Introduction}

Alcohol-water mixtures have long attracted researchers in chemistry, physics, and biosciences in connection with chromatography, solvent extraction, chemical synthesis, denaturation of proteins, etc. [1]. A number of thermodynamic and spectroscopic experiments have been carried out to understand their characteristic properties, such as anomalies of the heat of mixing [2], partial molar volume [3], NMR chemical shift [4], dielectric relaxation [5 - 7], specific heats [8], and ultrasonic attenuation [9], which have often been observed in the water-rich region of aqueous alcohol mixtures. These anomalous properties become prominent with increasing alkyl chain length of the alcohols.

Direct structural investigations on alcohol-water mixtures have been performed to reveal the structures by X-ray [10 - 12] and neutron diffraction [13 - 16],
Monte Carlo [17] and molecular dynamics simulation [18 - 20], mass spectrometry [10 - 12], smallangle X-ray [21 - 24] and neutron scattering [25, 26]; both microscopic and mesoscopic structural features of alcohol-water mixtures have been successfully elucidated and discussed in connection with anomalies in the physicochemical properties and microheterogeneity.

Recently, Egashira and Nishi have proved that lowfrequency Raman spectrometry is one of the powerful tools to investigate the clusters in ethanol-water mixtures [27]. It is interesting to apply this technique to other alcohol-water mixtures, where the balance between hydrophobicity and hydrophilicity changes with the size and shape of the alkyl chain.

In the present study we have measured the lowfrequency Raman spectra of aqueous solutions of methanol, ethanol, 1-propanol, 2-propanol, and tertbutyl alcohol as functions of alcohol mole fraction. 
Our aims are to show how the low-frequency Raman spectra reflect the structural characteristics such as microheterogeneity and transition of the solvent clusters in various alcohol-water mixtures as revealed by previous X-ray and neutron diffraction measurements.

\section{Experimental}

\subsection{Sample Preparation}

The alcohols were of spectrometric grade and used without further purification. Water was distilled twice under nitrogen atmosphere. All solutions were degassed before measurements. A flow-type cell of fused silica with 4 and $6 \mathrm{~mm}$ in inner and outer diameters, respectively, was used to keep the same beam alignment for different samples and hence to ensure constant background in the the spectra. The sample solutions were inserted with a peristaltic pump.

\subsection{Low-frequency Raman Spectroscopy}

The depolarized Rayleigh wing spectra $I(\bar{\nu})$ are the Fourier transform of the time-dependent macroscopic induced polarizability that can be written as a sum of point polarizabilites over all the $N$ molecules in the scattering volume [28 - 30]. In order to extract a quantity related to the vibrational density of states from the measured Raman spectrum, it is necessary to remove the intensity of the exciting laser line. The relation between the Rayleigh wing spectra and the intrinsic Raman scattering activity, $R(\bar{\nu})$, is given by

$$
R(\bar{\nu})=I(\bar{\nu})\left(\bar{\nu}_{0}-\bar{\nu}\right)^{-4} \bar{\nu}[1-\exp (-h c \bar{\nu} / k T)],
$$

where $\bar{\nu}_{0}$ is the frequency of the exciting laser line (in $\mathrm{cm}^{-1}$ ), $h$ the Planck constant and $c$ the velocity of light [27].

The Raman spectra in the low-frequency region $\left(20-300 \mathrm{~cm}^{-1}\right)$ were measured at $298 \mathrm{~K}$ on a Raman spectrometer (Joban Yvon T64000), equipped with a triple monochrometor and a CCD detector. The scattering light was measured at an angle of $90^{\circ}$ relative to the exciting laser beam provided by an argon-ion laser (Spectra Physics). The incident beam of $514.5 \mathrm{~nm}$ was used at a power of $500 \mathrm{~mW}$. The accumulation time per measurement was $120 \mathrm{~s}$, and the measurements were repeated twice to remove the peaks due to cosmic rays from the spectra of the samples.

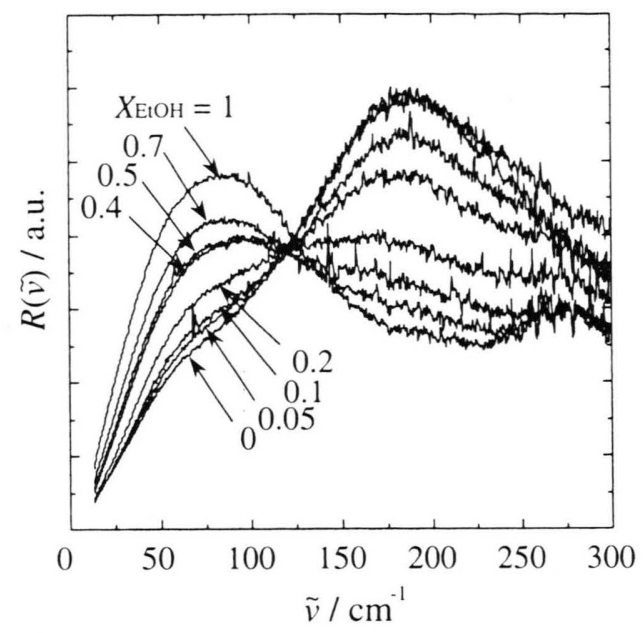

Fig. 1. $R(\bar{\nu})$ spectra of ethanol-water binary mixtures as functions of ethanol the mole fraction $x_{\mathrm{EtOH}}$.

\section{Results}

\subsection{Ethanol-water}

The ethanol-water mixtures were first measured to check the quality and reproducibility of the present low-frequency Raman spectra. Figure 1 shows the $R(\bar{\nu})$ spectra at various mole fractions of ethanol. These spectra agree well with those previously reported [27]. In the $R(\bar{\nu})$ spectrum of pure water $\left(x_{\mathrm{ETOH}}=0\right)$, two bands appear at 60 and $185 \mathrm{~cm}^{-1}$, which have been assigned to the collective modes of water molecules in the translational region [31]. The $185 \mathrm{~cm}^{-1}$ band has been assigned to the stretching motion of the $\mathrm{O}-\mathrm{O}$ hydrogen bond, whereas the weaker $60 \mathrm{~cm}^{-1}$ band is due to the O-O-O bending motion of the hydrogen-bonded trimer unit [27]. On the other hand, the $R(\bar{\nu})$ of pure ethanol $\left(x_{\mathrm{ETOH}}=1\right)$ reveals a trapezoidal band in the region of 40 $120 \mathrm{~cm}^{-1}$. Egashira and Nishi decomposed the band into two components at 60 and $110 \mathrm{~cm}^{-1}$, and assigned the latter to the intermolecular stretching motion of the O-O hydrogen bond on the basis of the far-infrared spectrum of pure ethanol [32, 33]. However, no definite assignment was made on the former component at $60 \mathrm{~cm}^{-1}$ [27]. Thus, hereafter, we tentatively regard the composite band at $83 \mathrm{~cm}^{-1}$ as one due to the intermolecular O-O stretching motion between ethanol molecules.

An isosbestic point has been observed at $121 \mathrm{~cm}^{-1}$, as in the literature [27]. The presence of the isosbestic point has been interpreted by Egashira and Nishi [27] 
in terms of a two-state model, i.e. two association states of individual water and ethanol aggregates without significant ethanol-water intermolecular interactions on a time scale in Raman spectroscopy. The $R(\bar{\nu})$ spectra of the mixtures have been expressed as a sum of those of the water and ethanol aggregates:

$$
R(\bar{\nu}, x)_{\mathrm{calc}}=w R(\bar{\nu}, 0)+a R(\bar{\nu}, 1)
$$

where $R(\bar{\nu}, x)_{\text {calc }}$ is the $R(\bar{\nu})$ spectrum of the mixture with an alcohol mole fraction $x, R(\bar{\nu}, 0)$ and $R(\bar{\nu}, 1)$ are those for pure water and pure alcohols, respectively, and $w$ and $a$ are the coefficients of the individual components. The coefficients $w$ and $a$ were independently varied to minimize the error squares sum $U$ by a linear least-squares fitting method,

$$
U=\Sigma\left[R(\bar{\nu}, x)_{\mathrm{obs}}-R(\bar{\nu}, x)_{\mathrm{calc}}\right]^{2} .
$$

The coefficients $w$ and $a$, and the sums are plotted as a function of $x$ in Figure 2. The present results are again in good agreement with those in [27]. The coefficients $w$ and $a$ have an inflection point at $x_{\mathrm{EtOH}} \approx 0.3$, which is slightly lower than the value (0.2) in [27]. Around this mole fraction, anomalies in thermodynamic data appear, and in previous X-ray diffraction measurements $[10,11]$ the predominant solvent clusters have been found to change from the tetrahedral water structure to the chain-like ethanol structure. Thus, the inflection points in the coefficients would indicate a structural transition of the solvent clusters responsible for thermodynamic anomalies and microheterogeneity. The sums of the coefficients become unity

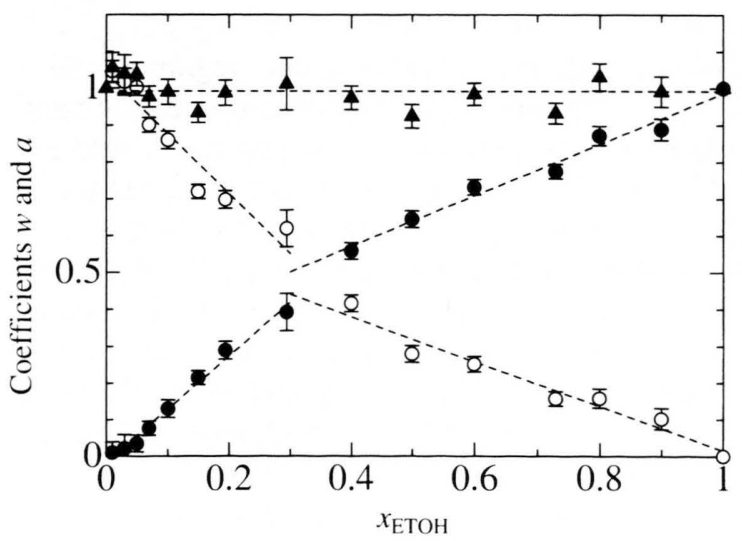

Fig. 2. Plot of the coefficients $w(0)$ and $a(\bullet)$, and the sums $(\boldsymbol{\Delta})$ as functions of the ethanol mole fraction $x_{\mathrm{EtOH}}$. within experimental uncertainty, independent of the ethanol concentration, as seen in Fig. 2, confirming the validity of the two-state model.

The present results show that the low-frequency spectra obtained in the present system are as suitable as those in the literature [27] to discuss the structural characteristics of the alcohol-water systems.

\subsection{1-propanol-water and 2-propanol-water}

Figure 3 shows the $R(\bar{\nu})$ of the 1-propanol-water mixtures at various alcohol mole fractions. In pure 1-propanol, a broad peak is observed at $69 \mathrm{~cm}^{-1}$, which is slightly sharper and lower in amplitude, and shifts to the lower wavenumber region than that for pure ethanol. As mentioned in the previous section, this band is assigned to the intermolecular stretching motion of the $\mathrm{O}-\mathrm{O}$ hydrogen bond between 1propanol molecules. This peak gradually decreases with decreasing alcohol concentration. An isosbestic point appears at $125 \mathrm{~cm}^{-1}$. Since the spectral features are very similar to that for the ethanol-water system, the two-state model has been applied to the spectra, followed by the least-squares fits. The final results are shown in Figure 4. Two inflection points in the coefficients have been observed at $x_{1-\mathrm{PrOH}} \approx$ 0.2 and 0.5 . These mole fractions are consistent with the solvent compositions at which anomalies in thermodynamic properties appear, e. g. the excess heat of mixing has a minimum at $x_{1-\mathrm{PrOH}} \approx 0.1$ and a maximum at $x_{1-\mathrm{PrOH}} \approx 0.6$ in 1-propanol-water mixtures.

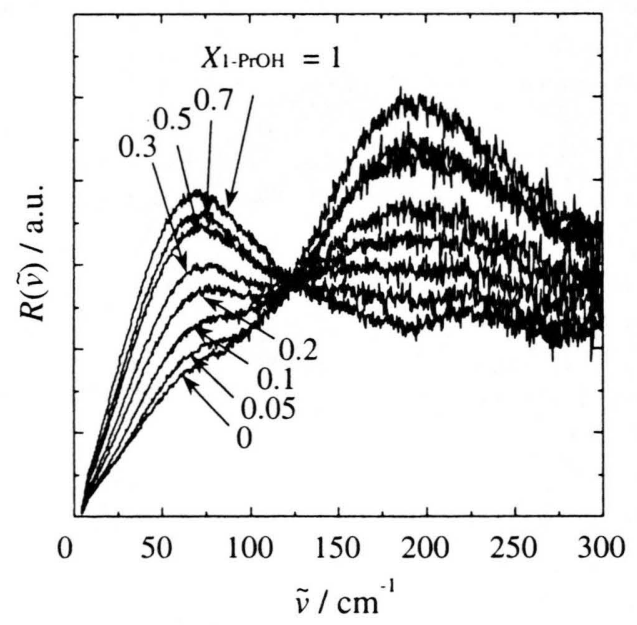

Fig. 3. $R(\bar{\nu})$ spectra of 1-propanol-water binary mixtures as functions of the 1-propanol mole fraction $x_{1-\mathrm{PrOH}}$. 


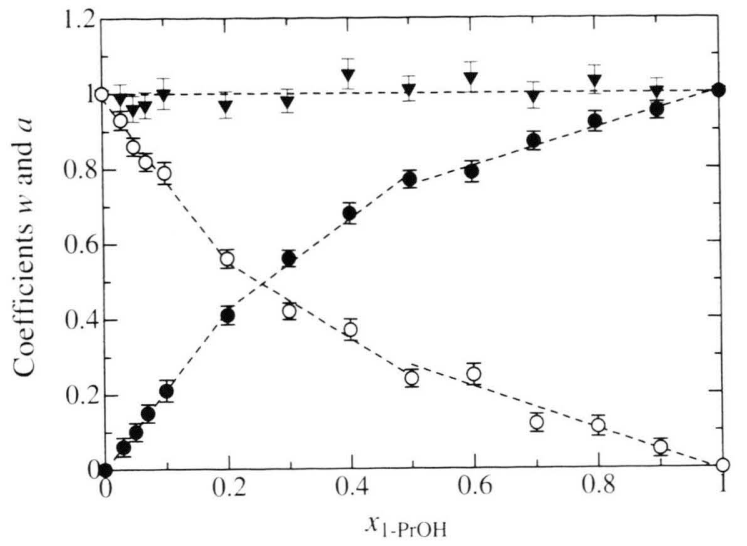

Fig. 4. Plot of the coefficients $w(0)$ and $a(\bullet)$, and the sums $(\boldsymbol{\Lambda})$ as functions of the 1-propanol mole fraction $x_{1-\mathrm{PrOH}} \cdot$

The sums of the coefficients are almost unity over the whole range of alcohol concentration, again supporting the two-state model to describe the clusters involved in the mixtures. The inflection points would suggest the probable transition of the solvent clusters from the tetrahedral-like water to the alcohol aggregation, and microheterogeneity in 1-propanol-water mixtures.

It is interesting to see that the coefficients $a$ and $w$ change more rapidly with alcohol concentration in the 1-propanol-water mixtures than in the ethanol-water mixtures. The crossing point of each coefficient is located at $x_{1-\mathrm{PrOH}} \approx 0.25$, which slightly shifts to the water-rich region compared with the case $(\approx 0.3)$ for

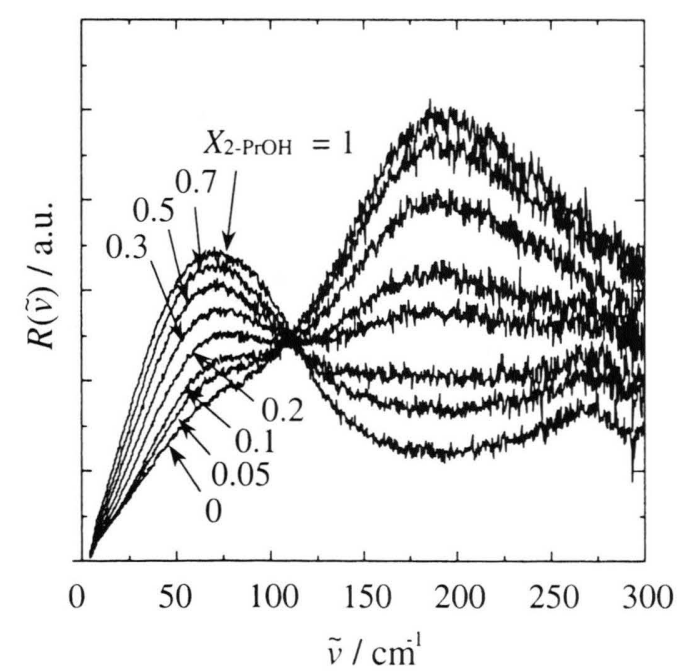

Fig. 5. $R(\bar{\nu})$ spectra of 2-propanol-water binary mixtures as functions of the 2-propanol mole fraction $x_{2-\mathrm{PrOH}}$.

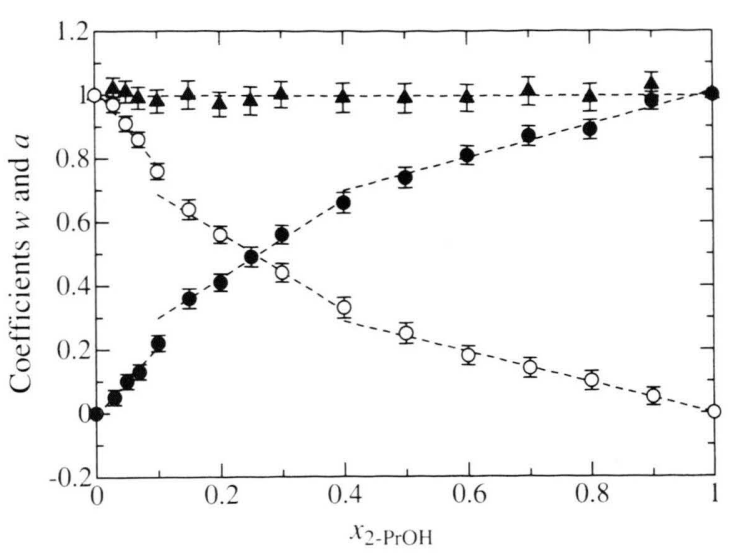

Fig. 6. Plot of the coefficients $w(0)$ and $a(\bullet)$, and the sums $(\boldsymbol{\Delta})$ as functions of the 2-propanol mole fraction $x_{2-\mathrm{PrOH}}$.

the ethanol-water mixtures. This shows that 1-propanol with its long hydrophobic alkyl group perturbs the water structure more effectively than ethanol.

Figure 5 shows the $R(\bar{\nu})$ of the 2-propanol-water mixtures at various alcohol mole fractions. The spectra are very similar to those for the 1-propanol-water mixtures, except that an isosbestic point appears at $111 \mathrm{~cm}^{-1}$, which is lower by $14 \mathrm{~cm}^{-1}$ in wavenumber than in the 1-propanol-water mixtures. The results of the two-state model analysis are shown in Figure 6 . The crossing point of each coefficient occurs at $x_{2 \text {-PrOH }} \approx 0.25$, similar to the case of the 1 -propanol-water mixtures. The sum of both coefficients is almost unity over the whole range of concentration. Two inflection points are observed at $x_{2-\mathrm{PrOH}} \approx 0.2$ and 0.4 as in the 1-propanol-water mixtures.

\subsection{Methanol-water}

Figure 7 shows the $R(\bar{\nu})$ spectra at various mole fractions of methanol. In pure methanol, the intermolecular O-O band appears as a peak at $119 \mathrm{~cm}^{-1}$ and a shoulder at $65 \mathrm{~cm}^{-1}$. The amplitude in intensity is much higher than that for the other alcoholwater mixtures. In contrast to the other three aliphatic alcohol-water mixtures, an isosbestic point has not been observed in the methanol-water mixtures, although a quasi-isosbestic point appears at $141 \mathrm{~cm}^{-1}$ when $x_{\mathrm{MeOH}} \geq 0.4$. This finding suggests that the structure of the methanol-water mixtures is not characterized by a simple two-state model of individual water and methanol aggregates, and significant hydrogen bonding between water and methanol molecules 


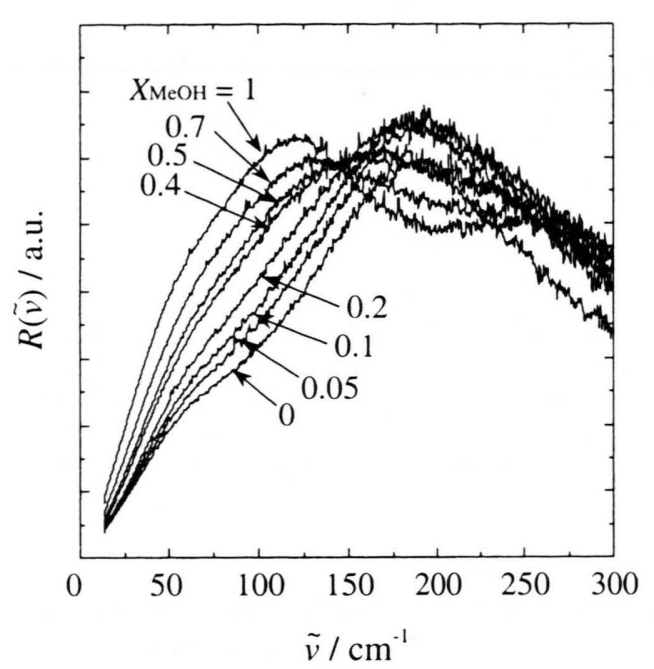

Fig. 7. $R(\bar{\nu})$ spectra of methanol-water binary mixtures as functions of the methanol mole fraction $x_{\mathrm{MeOH}}$.

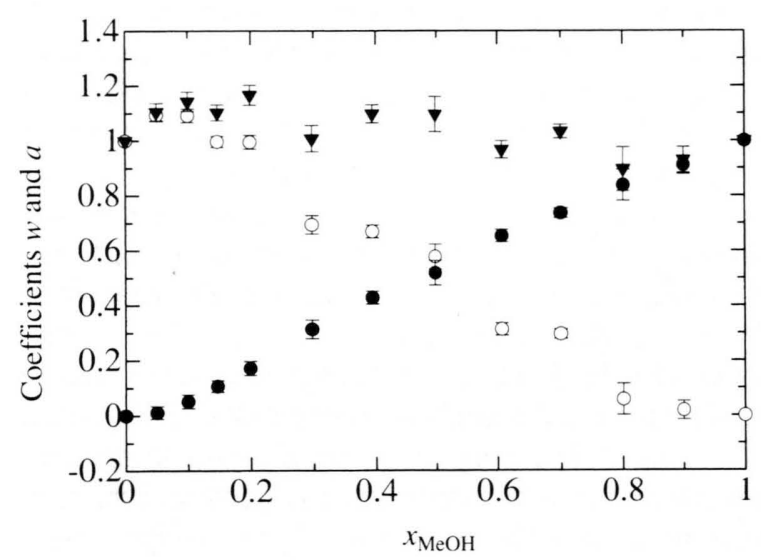

Fig. 8. Plot of the coefficients $w(\circ)$ and $a(\bullet)$, and the sums $(\boldsymbol{\Delta})$ as functions of the methanol mole fraction $x_{\mathrm{MeOH}}$.

is plausible, in particular, in the water-rich region $\left(x_{\mathrm{MeOH}} \leq 0.2\right)$.

The analysis of the two state model was made to see how the coefficients $w$ and $a$ would behave, compared with the cases of the ethanol-water and the propanol-water mixtures. The final results are shown in Figure 8. As is expected, the sums of the coefficients $w$ and $a$ deviate from unity at $x_{\mathrm{MeOH}} \leq 0.2$, but are almost unity within experimental uncertainties at $x_{\mathrm{MeOH}} \geq 0.4$. The decrease of $w$ and the increase of $a$ are more moderate in the methanol-water mixtures than those in aqueous mixtures of the other alcohols with longer alkyl chains. An inflection point in the coefficients appears to be at $x_{\mathrm{MeOH}}$ between 0.2 and 0.4 .
According to our previous X-ray diffraction experiment on methanol-water mixtures [12], transition of the predominant solvent clusters from the tetrahedral-like water cluster to the chain-like methanol aggregates takes place at $x_{\mathrm{MeOH}} \approx 0.3$, consistent with the composition of the inflection point estimated. The crossing point of each coefficient is situated at $x_{\mathrm{MeOH}} \approx 0.5$, which shifts to the alcohol-rich region, compared with the case for the other alcohol-water mixtures. All of these results show that perturbation of the water structure by methanol occurs less effectively than by the other alcohols with longer alkyl chains, and that microheterogeneity does not occur because of hydrogen bonding between methanol and water molecules, as previously discussed [12].

\subsection{TBA-water}

TBA is miscible with water in all proportions in spite of its large hydrophobic group, while the other isomers of butanol are less soluble in water. Figure 9 shows the $R(\bar{\nu})$ of the TBA-water mixtures. In pure TBA, a broad peak is observed at $64 \mathrm{~cm}^{-1}$, which is due to the hydroxyl O-O stretching of TBA. It should be noted that the wavenumber and the intensity of the peak are lowest among the aqueous alcohol mixtures investigated. A quasi-isosbestic point appears at $89 \mathrm{~cm}^{-1}$, where $x_{\mathrm{TBA}} \geq 0.05$.

A quantitative analysis of the two-state model was attempted to the $R(\bar{\nu})$ values in Figure 9. The final results are shown in Figure 10. Two inflection points

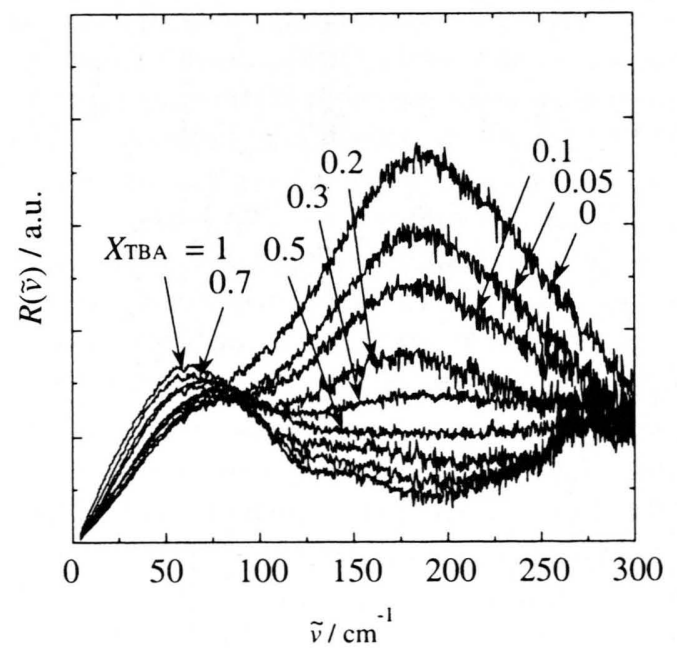

Fig. 9. $R(\bar{\nu})$ spectra of TBA-water binary mixtures as functions of the TBA mole fraction $x_{\mathrm{TBA}}$. 


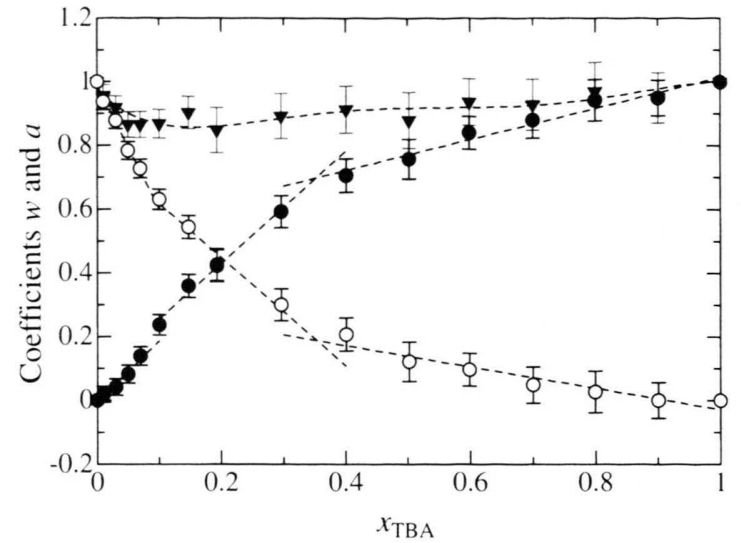

Fig. 10. Plot of the coefficients $w(0)$ and $a(\bullet)$, and the sums $(\boldsymbol{\Delta})$ as functions of the TBA mole fraction $x_{\mathrm{TBA}}$.

in the individual coefficients $w$ and $a$ are observed at $x_{\mathrm{TBA}} \approx 0.1$ and 0.35 , suggesting structural transition of the predominant clusters at these mole fractions. Unlike the other alcohol-water mixtures except for the methanol-water mixtures, the sum of the coefficients $a$ and $w$ has a broad minimum at $x_{\mathrm{TBA}} \approx 0.1$. This is due to the rapid decrement of the coefficient $w$ with increasing TBA concentration. This finding indicates that the bulk-like water structure is most effectively disrupted on addition of TBA among the alcohols investigated.

Here, it is interesting to note that the partial molar volume [3] and the enthalpy of mixing [2] for TBAwater mixtures are both largely negative at $0<x_{\mathrm{TBA}}<$ 0.06 and show an inflection point at $x_{\mathrm{TBA}} \approx 0.1$. A previous small-angle $\mathrm{X}$-ray scattering study on aqueous TBA solutions showed an enhancement of concentration fluctuations in the region $0.06<x_{\mathrm{TBA}}<0.3$ [22 - 24]. The mole fractions for the inflection points obtained from the present Raman spectra are consistent with those reported for the above macroscopic and mesoscopic characteristics of aqueous TBA solutions. In addition, as discussed previously, the inflection point at $x_{\mathrm{TBA}} \approx 0.1$ suggests a structural transition of predominant clusters in the TBA-water mixtures.

The structure of aqueous TBA solutions at around these mole fractions has recently been shown at the molecular level by an MD simulation [20] and by empirical potential structure refinement (epsr) studies combined with neutron diffraction experiments with H/D isotopic substitution [14 - 16]. According to the MD simulation of 0.02 and 0.08 mol fraction aqueous TBA solutions, TBA molecules associate to form
Table 1. Characteristic parameter values for aqueous solutions of methanol, ethanol, 1-proponal, 2-propanol, and TBA $^{\mathrm{a}}$.

\begin{tabular}{|c|c|c|c|c|c|}
\hline \multirow[b]{2}{*}{$\mathrm{ISP} / \mathrm{cm}^{-1}$} & \multicolumn{2}{|c|}{ methanol ethanol } & \multicolumn{2}{|c|}{ 1-propanol 2-propanol } & \multirow{2}{*}{$\begin{array}{c}\text { TBA } \\
(89)\end{array}$} \\
\hline & $(141)$ & 121 & 125 & 111 & \\
\hline$\nu(\mathrm{O}-\mathrm{O}) / \mathrm{cm}^{-1}$ & 65,119 & 83 & 69 & 72 & 64 \\
\hline$I_{\mathrm{a}} / I_{\mathrm{w}}$ & 1.04 & 0.79 & 0.79 & 0.69 & 0.43 \\
\hline IFP & $\sim 0.3$ & $\sim 0.3$ & $\sim 0.2,0.5$ & $\sim 0.2,0.4$ & $\sim 0.1,0.35$ \\
\hline
\end{tabular}

${ }^{a}$ ISP: isosbestic point, $\nu(\mathrm{O}-\mathrm{O})$ : wavenumber of the $\mathrm{O}-\mathrm{O}$ stretching vibration between alcohol molecules, $I_{\mathrm{a}} / I_{\mathrm{w}}$ : ratio of the peak intensity of the $\nu(\mathrm{O}-\mathrm{O})$ band for alcohol $\left(I_{\mathrm{a}}\right)$ to that for water $\left(I_{\mathrm{w}}\right)$, IFP: inflection point in the coefficients $w$ and $a$. The parentheses in ISP show the quasi-isosbestic point.

clusters of three to four molecules at a time scale of a few $\mathrm{ps}$ in the $x=0.08$ solution, but there is virtually no association in the $x=0.02$ solution. Concerning the water structure, at lower concentration TBA does not significantly affect the water structure, but, in the $x=0.08$ solution the local tetrahedral-like correlations are enhanced compared to that of pure water.

The epsr simulation study on $x=0.06,0.11$, and 0.16 aqueous TBA solutions has also shown the domination of nonpolar methyl group to methyl group direct contacts rather than via polar interactions of their hydroxyl groups in the $x=0.06$ solution. Hydrogen bonding between TBA molecules is absent even in the $x=0.16$ solution, although it has been found in pure TBA [15]. These results agree with the finding from the present Raman band due to the O-O stretching motion. The epsr study has also shown that the enhancement of the water-water structural correlation originates from the second neighbor distance rather than the first neighbor interations.

NMR relaxation measurements on aqueous TBA solutions under high pressures ( $200 \mathrm{MPa}$ ) have also suggested that in solutions of 4 to 5 mole $\%$ the hydration shell collapses and the interaction between water and the hydroxyl group of TBA is predominant [34].

\section{Discussion}

Table 1 summarizes significant parameters for all alcohol-water mixtures investigated. For pure alcohols the wavenumber, $\nu(\mathrm{O}-\mathrm{O})$, of the composite band due to the O-O stretching motion between alcohol molecules decreases in the order of methanol > ethanol > 1-propanol $\approx 2$-propanol $>$ TBA. Moreover, the ratio, $I_{\mathrm{a}} / I_{\mathrm{w}}$, of the peak intensities of the $\nu(\mathrm{O}-\mathrm{O})$ band for alcohols to that for water also decreases in 
the same order. These findings suggest that the hydrogen bond between alcohol molecules is weakened in the order of methanol $>$ ethanol $>1$-propanol $\approx$ 2-propanol > TBA.

The mole fraction for the (first) inflection points decreases with increasing the alkyl chain length of $n$-alcohols and is lowest for the bulky TBA. Since the first inflection point corresponds to the transition of the predominant clusters from the tetrahedral-like water to the alcohol aggregates, these results shows that the longer the alkyl chains of the alcohols the faster the water structure is disrupted.

As to the two isomers of propanol, Table 1 shows subtle differences in characteristic parameters of the two isomers. According to a small-angle neutron scattering study of aqueous solutions of 1-propanol $\left(x_{1-\mathrm{PrOH}}=0.114\right)$ and 2-propanol $\left(x_{2-\mathrm{PrOH}}=0.134\right)$ in $\mathrm{D}_{2} \mathrm{O}$ [25], the radius of gyration was determined as 10.2 and $5.20 \AA$, respectively, at $25^{\circ} \mathrm{C}$, suggesting that the concentration fluctuations are larger in the 1propanol aqueous solution than in the $2-\mathrm{PrOH}$ aqueous solution. The mesoscopic ordering in aqueous propanol solutions might be caused by the different position of the hydroxyl group of propanol.

The inflection points in the coefficients $w$ and $a$ appear to associate with the anomalous behavior of the thermodynamic data of these systems, the structure transition of predominant clusters in the solutions, and microheterogeneity in the systems. According to a molecular dynamics study on dilute aqueous solutions of a series of $n$-alcohols from methanol to butanol (a system of one alcohol and 255 water molecules) [19], it has been found that the static structure of water around the hydrophobic end of the alcohols is essen-

[1] F. Franks, J. E. Desnoyers, In Water Science Reviews 1, ed. F. Franks, University Press, Cambridge 1985, p. 171, and the references therein.

[2] F. Franks and D. J. G. Ives, Q. Rev. Chem. Soc. 20, 1 (1966).

[3] K. Nakanishi, Bull. Chem. Soc. Japan. 33, 793 (1960).

[4] A. Coccia, P. L. Inodovia, F. Podo, and V. Viti, Chem. Phys. 7, 30 (1975).

[5] S. Mashimo, S. Kuwabara, S. Yagihara, and K. Higashi, J. Chem. Phys. 90, 3292 (1989).

[6] N. Asaka, N. Shinyashiki, T. Umehara, and S. Mashimo, J. Chem. Phys. 93, 8273 (1990).

[7] S. Mashimo, T. Umehara, and H. Redlin, J. Chem. Phys. 95, 6257 (1991). tially the same as that found in bulk water; in particular, there was no evidence of a clathrate-like cage around the hydrophobic end of the alcohol. Some enhancement of the water structure was found in the vicinity of the alcohol hydroxyl group, with the hydrogen bonding network being closer to tetrahedral in the solvation shell than in bulk water. Thus, the microheterogeneity requires a certain amount of alcohol molecules to form aggregates, which has been shown as the inflection points in the present study.

\section{Conclusion}

The low-frequency Raman spectra measured on aqueous solutions of a series of mono-hydroxyl alcohols have proved to be useful in giving information on the structure of hydrogen-bonded clusters in the mixtures. The composite bands in the region of 70 - $120 \mathrm{~cm}^{-1}$ for pure alcohols, assigned to the O-O stretching vibration of the hydrogen bonds between alcohol molecules, have suggested that the hydrogen bond is weakened in the order of methanol $>$ ethanol $>1$-propanol $\approx 2$-propanol $>$ TBA. The Raman spectra of alcohol-water mixtures such as ethanol, 1-propanol, and 2-propanol, in which microheterogeneity occur, have a clear isosbestic point to support the two-state model of individual alcohol aggregates and water clusters. The two-state model analysis of the low-frequency Raman spectra as functions of alcohol mole fraction has given inflection points at characteristic alcohol mole fractions, which correspond to anomalies in thermodynamic data and structural transitions of the predominant clusters in aqueous alcohol mixtures.

[8] G. Roux, G. Perron, and J. E. Desnoyers, J. Phys. Chem. 82, 966 (1978).

[9] M. Brai and U. Kaatze, J. Phys. Chem. 96, 8946 (1992).

[10] N. Nishi, S. Takahashi, S. Matsumoto, A. Tanaka, K. Muraya, T. Takamuku, and T. Yamaguchi, J. Phys. Chem. 99, 462, (1995).

[11] M. Matsumoto, N. Nishi, T. Furusawa, M. Saita, T. Takamuku, M. Yamagami, and T. Yamaguchi, Bull. Chem. Soc. Japan. 68, 1775 (1995).

[12] T. Takamuku, T. Yamaguchi, M. Asato, M. Matsumoto, and N. Nishi, Z. Naturforsch. 55a, 513 (2000). 
[13] A. K. Soper and J. L. Finney, Phys. Rev. Lett. 71, 4346 (1993).

[14] D. T. Bowron, J. L. Finney, and A. K. Soper, J. Phys. Chem. B 102, 3551 (1998).

[15] D. T. Bowron, J. L. Finney, and A. K. Soper, Mol. Phys. 93, 531 (1998).

[16] D. T. Bowron, J. L. Finney, and A. K. Soper, J. Chem. Phys. 114, 6203 (2001).

[17] K. Nakanishi, K. Ikari, S. Okazaki, and H. Touhara, J. Chem. Phys. 80, 1656 (1984).

[18] G. Pálinkás and I. Bakó, Z. Naturforsch. 46a, 95 (1991).

[19] J. Fidler and P. Rodger, J. Phys. Chem. B 103, 7695 (1999)

[20] P. G. Kusalik, A. P. Lyubartsev, D. L. Bergman, and A. Laaksonen, J. Phys. Chem. B 104, 9533 (2000).

[21] K. Nishikawa, Y. Kodera, and T. Iijima, J. Phys. Chem. 91, 3694(1987).

[22] K. Nishikawa, H. Hayashi, and T. Iijima, J. Phys. Chem. 93, 6559 (1989).

[23] H. Hayashi, K. Nishikawa, and T. Iijima, J. Phys. Chem. 94, 8334 (1990).
[24] K. Nishikawa and T. Iijima, J. Phys. Chem. 97, 10824 (1993).

[25] G. D'Arrigo and J. Teixeira, J. Chem. Soc. Faraday Trans. 86, 1503 (1990).

[26] K. Yoshida, M. Misawa, K. Maruyama, M. Imai, and M. Furusaka, J. Chem. Phys. 113, 2343 (2000).

[27] K. Egashira and N. Nishi, J. Phys. Chem. B 102, 4054 (1998).

[28] P. A. Madden and R. W. Impey, Chem. Phys. Lett. 123, 502 (1986).

[29] R. W. Impey, P. A. Madden, and I. R. McDonald, Mol. Phys. 46, 513 (1982).

[30] G. J. Safford, P. S. Leung, A. W. Naumann, and P. C. Schaffer, J. Chem. Phys. 50, 4444 (1969).

[31] S. Sastry, H. E. Stanley, and F. Sciortino, J. Chem. Phys. 100, 5361 (1994).

[32] R. F. Lake and H. W. Thompson, Proc. Roy. Soc. London A, 291, 469 (1966).

[33] J. K. Vij, C. J. Reid, and M. W. Evans, Chem. Phys. Lett. 92, 528 (1982).

[34] K. Yoshida, K. Ibuki, and M. Ueno, J. Chem. Phys. 108, 1360 (1998). 